\title{
HYPERBOLIC TRAVELLING FRONTS
}

\author{
by K. P. HADELER
}

(Received 10th June 1986)

\section{Introduction}

Since the work of R. A. Fisher [2] and Kolmogorov, Petrovskij and Piskunov [7] (see [6] for further references) the problem of travelling fronts in reaction-diffusion equations has been extensively studied. For the equation

$$
u_{t}=u_{x x}+F(u)
$$

with $F(0)=F(1)=0$ a travelling front is a solution

$$
u(t, x)=\phi(x-c t),
$$

where the function of one variable $\phi$ is decreasing and satisfies $\phi(-\infty)=1, \phi(+\infty)=0$. The function $\phi$ describes the shape of the front and the constant $c$ is the speed of propagation. There are two main types of the problem. In the all-positive case, where the function $F$ satisfies

$$
F(u)>0 \text { for } 0<u<1 \text {, }
$$

there is a half-line $\left[c_{0}, \infty\right), c_{0}>0$, of speeds. For each $c \in\left[c_{0}, \infty\right)$ there is, up to translation, a unique travelling front. Fronts for different $c$ can be distinguished by the rate of decay towards $+\infty$. In the threshold case, where $F$ has the property, for some $\gamma \in(0,1)$

$$
\begin{aligned}
& F(u)<0 \text { for } \quad 0<u<\gamma \\
& F(u)>0 \text { for } \quad \gamma<u<1,
\end{aligned}
$$

there is a unique speed $c_{0}$ with a travelling front, which is unique up to translation. In this case the sign of $c_{0}$ is determined by the sign of the integral $\int_{0}^{1} F(u) d u$.

The front solutions $u$ to equation (1) can be interpreted in several ways. In Fisher's model there are two types of individuals (or genes) $A$ and $a$ in a population, and $u(t, x)$ measures the frequency of type $A$ at the space point $x$ at time $t$. Type $A$ is advantageous and moves from $x \rightarrow-\infty$ to $x \rightarrow+\infty$. In the ecological interpretation of equation (1) as a Verhulst equation with diffusion the value $u(t, x)$ is the population density at point $x$ at time $t$. The population moves from left to right into a sparsely populated biotope. In 
McKean's interpretation [8] the function $u(t, x)$ is related to a branching process with Brownian motion. At each time $t$ there are finitely many particles which perform Brownian motion and split according to a branching process. Let $u(t, x)$ be the probability that at time $t$ not all particles are located to the left of $x$. Then $u$ satisfies an equation (1). In Fisher's model the equation describes the bulk of the population, in McKean's interpretation it describes the most advanced particle.

In $[4,5]$ equation $(1)$ has been generalized to an equation of the form

$$
m(u) u_{t}=\left(k(u) u_{x}\right)_{x}+f(u) .
$$

The essential feature is the density-dependent diffusion rate $k$, whereas the "densitydependent capacity" $m$ does not admit a convincing physical interpretation. However, this coefficient is useful in the following treatment of the hyperbolic case. Of course, the general partial differential equation (4) cannot be transformed into an equation of the form (1). However, in [5] I have shown that the problem for travelling front solutions of equation (4) can be reduced to a similar problem for equation (1). This reduction greatly simplifies the existence problem for travelling fronts, since the essential features of the three parameter functions $f, k, m$ are incorporated into a single function.

In a forthcoming paper S. Dunbar and $\mathrm{H}$. Othmer [1] consider a Poisson migration process with branching. A newborn particle moves with constant velocity to the right, say. It stays in this state with exponential holding time. If it leaves this state, then it reverses direction and starts a similar motion in the opposite direction. Also the particle may split, in the simplest case into two daughters. Each of the newborns chooses its direction of movement with probability $1 / 2$. At any time there are finitely many particles which have specified location, direction of movement, and age. As in McKean's approach, Dunbar and Othmer consider the function $u(t, x)$ which is the probability that at time $t$ not all particles are to the left of $x$. They show that $u(t, x)$ satisfies a hyperbolic equation of the general form

$$
\varepsilon^{2} u_{t t}+g(u) u_{t}=\left(k(u) u_{x}\right)_{x}+f(u) .
$$

In the actual application [1] one has $k \equiv$ constant, and $f, g$ are quadratic polynomials or, more general, certain probability generating functions. On comparing the present equations with the equations of Dunbar and Othmer one has to observe the transformation $u \rightarrow 1-u$.

The question arises whether equation (5) admits travelling fronts and how the speeds can be determined. Looking at equation (5) as a singular perturbation of Fisher's equation or as a perturbed wave equation one can conjecture propagation of signals with approximate speed $1 / \varepsilon$ or propagation of shaped signals with a speed essentially given by Fisher's equation. Both views are justified in some sense.

A direct discussion of the phase-plane problem resulting from equation (5) is rather inconvenient. But the reduction trick introduced in [5] works for the hyperbolic case as well. In the next sections I introduce a simplifying transformation for general parameter functions $g, k, u$. It leads to a rather complete description for the case of a constant or a monotone function $k$. The reduction trick for parabolic problems has been rediscovered by Engler [9]. For further references on travelling fronts see also [6, 10]. 


\section{Existence of fronts}

For definiteness I require $g, k, f \in C^{2}[0,1]: g(u)>0, k(u)>0$ for $u \in[0,1] ; f(0)=f(1)=0$, $f^{\prime}(0) f^{\prime}(1)<0, f(u)>0$ for $u \in(0,1)$.

The first result concerns the case $k \equiv 1$ motivated by the problem of Dunbar and Othmer [1].

Theorem 1. Let $k \equiv 1$. Then for the hyperbolic equation (5) there is a continuum $\left[c_{h}, 1 / \varepsilon\right)$ of speeds. To each $c \in\left[c_{h}, 1 / \varepsilon\right)$ there is, up to translation, a unique travelling front. The minimal speed $c_{h}$ is given by

$$
c_{h}=\frac{c_{F}}{\sqrt{1+\varepsilon^{2} c_{F}^{2}}}
$$

where $c_{F}$ (Fisher speed) is the minimal speed of the problem (1) with

$$
F(u)=\frac{1}{\kappa} \frac{f\left(G^{-1}(u)\right)}{g\left(G^{-1}(u)\right)}
$$

where

$$
\kappa=\int_{0}^{1} g(y) d y, \quad G(u)=\frac{1}{\kappa} \int_{0}^{u} g(y) d y .
$$

Formula (6) can be interpreted as follows. The minimal speed of the hyperbolic problem is always less than the "natural hyperbolic speed" $1 / \varepsilon$, and also less than $c_{F}$. If $f, g$ are fixed then $c_{h} \approx c_{F}$ for small $\varepsilon$ and $c_{h} \approx 1 / \varepsilon$ for large $\varepsilon$.

Theorem 2. Assume that $k$ is a strictly increasing function. There is a number $\varepsilon^{*}$ such that for each $\varepsilon \in\left(0, \varepsilon^{*}\right)$ there is a continuum of speeds of travelling fronts $\left[c_{h}(\varepsilon), \alpha_{1} / \varepsilon\right)$, where $\alpha_{1}=\sqrt{k(0)}$. For $\varepsilon>\varepsilon^{*}$ there are no proper travelling fronts, but a unique shock.

Theorem 3. Assume that $k$ is a strictly decreasing function. There is a number $\varepsilon^{*}$ such that for each $\varepsilon \in\left(0, \varepsilon^{*}\right)$ there is a continuum of speeds of travelling fronts $\left[c_{h}(\varepsilon), \alpha_{1} / \varepsilon\right)$, where $\alpha_{1}=\sqrt{k(1)}$. For $\varepsilon>\varepsilon^{*}$ the front splits in two layers travelling at different speeds.

Example. Let $g(u) \equiv g>0$ constant

$$
\begin{array}{ll}
k(u)=p+q u, & p>0 \\
f(u)=a u(1-u), & a>0 .
\end{array}
$$

If $q>0$ then

$$
\varepsilon^{*}=g \sqrt{\frac{2 p}{q a}} ; \quad c_{h}=\sqrt{\frac{4 a p}{g^{2}+4 a \varepsilon^{2}}}, \quad 0<\varepsilon<\varepsilon^{*}
$$


If $-p<q<0$ then

$$
\varepsilon^{*}=\frac{g}{2} \sqrt{\frac{p-(q)}{a(q)}} ; \quad c_{h}=\sqrt{\frac{4 a p}{g^{2}+4 a \varepsilon^{2}}}, \quad 0<\varepsilon<\varepsilon^{*} .
$$

\section{Proofs}

The expression (2), introduced into equation (5), yields the ordinary differential equation of second order

$$
\varepsilon^{2} c^{2} \phi^{\prime \prime}-c g(\phi) \phi^{\prime}=\left(k(\phi) \phi^{\prime}\right)^{\prime}+f(\phi)
$$

where ' denotes differentiation with respect to $\xi=x-c t$.

Again put

$$
u=\phi, \quad v=\phi^{\prime},
$$

and denote the independent variable by $t$. Then (9) becomes a first order system

$$
\begin{gathered}
\dot{u}=v \\
\left(k(u)-\varepsilon^{2} c^{2}\right) \dot{v}=-c g(u) v-k^{\prime}(u) v^{2}-f(u) .
\end{gathered}
$$

Now the reducing transformation is applied.

Define

$$
k_{0}=\min _{0 \leqq u \leqq 1} k(u)
$$

For the moment assume that

$$
\varepsilon^{2} c^{2}<k_{0}
$$

Define the function

$$
K(u) \equiv k(u)-\varepsilon^{2} c^{2} .
$$

Then

$$
K^{\prime}(u)=k^{\prime}(u)
$$

Now the system (10) reads

$$
\begin{gathered}
\dot{u}=v, \\
\dot{v}=-c \frac{g(u)}{K(u)} v-\frac{K^{\prime}(u)}{K(u)} v^{2}-\frac{f(u)}{K(u)} .
\end{gathered}
$$


Define

$$
\begin{gathered}
\kappa=\int_{0}^{1} g(y) d y, \\
G(u)=\frac{1}{\kappa} \int_{0}^{u} g(y) d y .
\end{gathered}
$$

The new dependent variables are

$$
\begin{gathered}
\tilde{u}=G(u), \\
\tilde{v}=\frac{1}{\kappa} K(u) v .
\end{gathered}
$$

Then

$$
\begin{gathered}
\dot{\tilde{u}}=G^{\prime}(u) \dot{u}=\frac{1}{\kappa} g(u) \dot{u}=\frac{1}{\kappa} g(u) v \\
=\frac{1}{\kappa} g(u) \frac{\kappa}{K(u)} \tilde{v}=\frac{g(u)}{K(u)} \tilde{v}, \\
\dot{v}=\frac{1}{\kappa} K^{\prime}(u) \dot{u} v+\frac{1}{\kappa} K(u) \dot{v} \\
=\frac{1}{\kappa} K^{\prime}(u) v^{2}+\frac{1}{\kappa} K(u)\left[-\frac{c g(u)}{K(u)} v-\frac{K^{\prime}(u)}{K(u)} v^{2}-\frac{f(u)}{K(u)}\right] \\
=-\frac{c}{\kappa} g(u) \frac{\kappa}{K(u)} \tilde{v}-\frac{1}{\kappa} f(u) .
\end{gathered}
$$

Hence

$$
\begin{gathered}
\tilde{u}=\frac{g(u)}{K(u)} \tilde{v}, \\
\tilde{v}=-c \frac{g(u)}{K(u)} \tilde{v}-\frac{1}{\kappa} f(u) .
\end{gathered}
$$

Next rescale the independent variable along trajectories. This transformation does not affect $c$,

$$
\begin{gathered}
\dot{u}=\tilde{v} \\
\dot{v}=-c \tilde{v}-\left[\tilde{k}(\tilde{u})-\varepsilon^{2} c^{2}\right] \tilde{f}(\tilde{u})
\end{gathered}
$$


where

$$
\left.\begin{array}{c}
f(\tilde{u})=\frac{1}{\kappa} \frac{f(u)}{g(u)} \\
\tilde{k}(\tilde{u})=k(u)
\end{array}\right\} \quad u=G^{-1}(\tilde{u}) .
$$

For the discussion of the problem (19) I consider the problem

$$
\begin{gathered}
\dot{u}=v, \\
\dot{v}=-c v-\left[\tilde{k}(u)-\alpha^{2}\right] \tilde{f}(u)
\end{gathered}
$$

and determine the speeds $c$ depending on $\alpha$.

First consider the case $k \equiv 1$. Then $\widetilde{k} \equiv 1$, and equation (21) becomes

$$
\begin{gathered}
\dot{u}=v \\
\dot{v}=-c v-\left[1-\alpha^{2}\right] \widetilde{f}(u) .
\end{gathered}
$$

From the known results on Fisher's equation it follows that there is a half-line of speeds $\left[c_{0}(\alpha), \infty\right)$ for each $\alpha \in[0,1)$ (see $[3,4]$ ). It is trivial that

$$
c_{0}(\alpha)=\sqrt{1-\alpha^{2}} c_{F},
$$

where $c_{F}=c_{0}(0)$ is the minimal speed of Fisher's equation (1) with $F(u)=\widetilde{f}(u)$. Now the minimal speed $c_{h}$ is given by $c_{h}=\alpha_{0} / \varepsilon$, where $\alpha_{0}$ is the solution of $\alpha=c_{0}(\alpha) \varepsilon$, hence (6) follows. For each $c \in\left[c_{h}, 1 / \varepsilon\right)$ there is a travelling front. Hence Theorem 1 is proved.

Next consider the case of a general (positive) function $k$. Again consider the equation (21). For the moment drop the hypothesis (12). For small $\alpha$ there is a spectrum $\left[c_{0}(\alpha), \infty\right)$. If $\alpha$ is increased, the function $k(u)-\alpha^{2}$ changes sign, and a complicated pattern of fronts appears (most of these will be unstable).

However, consider the case where the (original) function $k$ is strictly increasing. If $\alpha$ increases from 0 to $+\infty$ then the function

$$
\tilde{f}(u)=\left[\tilde{k}(u)-\alpha^{2}\right] \tilde{f}(u)
$$

undergoes a regular transition between different types (positive, threshold, negative) at the critical values

$$
\alpha_{1}=(k(0))^{1 / 2}, \quad \alpha_{2}=\left(\int_{0}^{1} k(y) d y\right)^{1 / 2}, \quad \alpha_{3}=(k(1))^{1 / 2} .
$$

Thus for $\alpha \in\left(0, \alpha_{1}\right)$ problem (21) admits a half-line [ $\left.c_{0}(\alpha), \infty\right)$ of speeds, for $\alpha \in\left(\alpha_{1}, \alpha_{2}\right)$ there is a single positive speed $c_{0}(\alpha)$, for $\alpha \in\left(\alpha_{2}, \alpha_{3}\right)$ there is a single negative speed, and 
for $\alpha \in\left(\alpha_{3}, \infty\right)$ there is a half-line of negative speeds. The speed $c_{0}(\alpha)$ is a continuous and nonincreasing function of $\alpha$ [4].

For every $\varepsilon>0$ the equation $\alpha=c_{0}(\alpha) \varepsilon$ has a unique solution $\alpha^{*}(\varepsilon) \in\left(0, \alpha_{2}\right)$. If $\varepsilon$ increases from 0 to $+\infty$ then $\alpha^{*}(\varepsilon)$ increases from 0 to $\alpha_{2}$. There is a unique $\varepsilon^{*}$ such that $\alpha^{*}\left(\varepsilon^{*}\right)=\alpha_{1}$. For $\varepsilon \in\left(0, \varepsilon^{*}\right)$ the corresponding function $\hat{f}$ has no sign change in $(0,1)$, for $\varepsilon>\varepsilon^{*}$ this function has a single sign change. The minimal (resp. unique) speed of problem (19) is $c_{h}=\alpha^{*}(\varepsilon) / \varepsilon$.

For $\varepsilon \in\left(0, \varepsilon^{*}\right)$ the corresponding function $\hat{f}$ has no sign change in $(0,1)$. Hence the original problem (5) has a continuum of speeds $\left[c_{h}, \alpha_{1} / \varepsilon\right.$ ). For $\varepsilon>\varepsilon^{*}$ problem (19) has a unique speed $c_{h}$, and the transformation between (19) and (10) is singular.

Formally the result of the reverse transformation is two functions $\left(u_{+}, v_{+}\right),\left(u_{-}, v_{-}\right)$ defined on a half-line $-\infty<t<0$ where $u_{+}$decreases from 1 to some $u_{0}$ and $u_{-}$ increases from 0 to $u_{0}$. Here $u_{0}$ is defined by $k\left(u_{0}\right)=c^{2} \varepsilon^{2}$. At $t=0$, i.e. at $u=u_{0}$, both branches join with a vertical tangent, $v_{ \pm} \rightarrow \mp \infty$.

Now consider the opposite case, where the positive function $k$ is strictly decreasing. Then the function $\hat{f}(u)$ is of type I for $\alpha<\alpha_{1}=(k(1))^{1 / 2}$. For $\alpha>\alpha_{1}$ the function $f(u)$ assumes negative values near $u=1$, there is no front of problem (22) connecting $u=1$ and $u=0$, but a value $u_{0} \in(0,1)$ and two fronts connecting $u_{0}$ to 1 and $u_{0}$ to 0 . Again, for $0<\alpha<\alpha_{1}$ let $c_{0}(\alpha)$ be the minimal speed of problem (22). If $\varepsilon<\alpha_{1} / c_{0}\left(\alpha_{1}\right)$ then the minimal speed of the hyperbolic problem is $\alpha^{*}(\varepsilon) / \varepsilon$ where $\alpha^{*}(\varepsilon)$ is the unique solution of the equation $c_{0}(\alpha) \varepsilon=\alpha$. For $\varepsilon$ approaching the value $\alpha_{1} / c\left(\alpha_{1}\right)$ the fronts split into an upper part with values in $\left(u_{0}, 1\right)$ moving to the left and a lower part moving to the right.

Thus, in the two cases of strictly monotone $k$, the problem behaves like the parabolic problem for small $\varepsilon$, at a certain critical $\varepsilon$ the fronts split.

In [3] the minimal resp. unique speed for Fisher's equation (1) has been explicitly determined for an arbitrary cubic function $f$. I retain the notation of [3].

If $F$ is nonnegative,

$$
F(u)=u(1-u)(1+v u), \quad-1 \leqq v<+\infty
$$

then

$$
c_{F}=\left\{\begin{array}{lc}
2 & -1 \leqq v<2 \\
\frac{v+2}{\sqrt{2 v}} & 2 \leqq v .
\end{array}\right.
$$

If $F$ has a sign change,

$$
F(u)=u(1-u)(u-\mu), \quad 0<\mu<1
$$

then

$$
C_{F}=1 / \sqrt{2}-\mu \sqrt{2}
$$


These explicit expressions lead to a better understanding of the phenomena related to nonconstant $k$. Consider equation (5) with

$$
\begin{aligned}
g(u) \equiv g>0 & \\
k(u)=p+q u, & p, q>0 \\
f(u)=a u(1-u), & a>0 .
\end{aligned}
$$

With this particular choice equation (21) assumes the form

$$
\begin{gathered}
\dot{u}=v \\
\dot{v}=-c v-\left(p+q u-\alpha^{2}\right) \frac{a}{g^{2}} u(1-u) .
\end{gathered}
$$

After elementary calculations one finds for $c(\alpha)$,

$$
\begin{gathered}
\alpha^{2}<p-q / 2: \quad c(\alpha)=2 \frac{\sqrt{a}}{g} \sqrt{p-\alpha^{2}} \\
p-q / 2<\alpha^{2}<p: \quad c(\alpha)=\frac{\sqrt{a}}{g} \frac{q+2\left(p-\alpha^{2}\right)}{\sqrt{2 q}} \\
p<\alpha^{2}<p+q: \quad c(\alpha)=\frac{\sqrt{a}}{g} \cdot\left(\sqrt{\frac{q}{2}}-\sqrt{\frac{2}{q}}\left(\alpha^{2}-p\right)\right) .
\end{gathered}
$$

Observe $c(\alpha)=0$ for $\alpha^{2}=p+q / 2$. In the first two cases the source term in (24) is of positive type, in the third case it is of threshold type.

From the equation $\varepsilon c(\alpha)=\alpha$ one finds the following explicit expressions for $c_{h}$

$$
\varepsilon^{2}<\frac{2 p-q}{4 q} \cdot \frac{g^{2}}{a}: c_{h}=\sqrt{\frac{4 a p}{g^{2}+4 a \varepsilon^{2}}}
$$

$$
\left.\begin{array}{l}
\frac{2 p-q}{4 q} \cdot \frac{g^{2}}{a}<\varepsilon^{2}<\frac{2 p}{q} \cdot \frac{g^{2}}{2} \\
\frac{2 p}{q} \cdot \frac{g^{2}}{a}<\varepsilon^{2}
\end{array}\right\}
$$

The appearance of different expressions for the speed reflects the two situations in (23a), which are distinguished by their phase planes. In type I the heteroclinic orbit enters a one-tangent node, in type II it enters the side manifold of a two-tangent node. In the present situation type I does not occur if $2 p<q$. Type I, II fronts correspond to a 
positive source function, type III to a threshold function. Nevertheless $c_{h}$ has the same analytic expression in cases II, III. The case $q \in(-p, 0)$ is treated similarly.

\section{REFERENCES}

1. S. Dunbar and H. Othmer, On a nonlinear hyperbolic equation describing transmission lines, cell movement, and branching random walks, in Nonlinear oscillations in biology and chemistry, H. G. ОтнмеR (Ed.) (Lecture Notes in Biomathematics, Springer-Verlag, 1986).

2. R. A. Fisher, The advance of advantageous genes, Ann. of Eugenics 7 (1937), 355-369.

3. K. P. HADELER and F. Rothe, Travelling fronts in nonlinear diffusion equations, J. Math. Biol. 2 (1975), 251-263.

4. K. P. HADELER, Travelling fronts and free boundary value problems, in Numerical treatment of free boundary value problems (Oberwolfach Conference 1980), J. ALbrecht, L. Collatz and K. H. HofFMANN (Eds.) (Birkhäuser-Verlag, 1981), 90-107.

5. K. P. HADELER, Free boundary problems in biological models, in Free boundary problems: Theory and applications, Vol. II (Montecatini Conference 198I), A. FASANO and M. Primicerio (Eds.) (Pitman, 1983), 664-671.

6. K. P. Hadeler, Spread and age structure in epidemic models, in Perspectives in mathematics, W. JäGER et al. (Eds.) (Birkhäuser-Verlag, 1984).

7. A. Kolmogorov, I. Petrovskis and N. Piskunov, Etude de l'equation de la diffusion avec croissance de la quantité de la matière et son application a une problème biologique, Bull. Univ. Moscou, Ser. Int., Sec. A 1, 6 (1937), 1-25.

8. H. P. McKean, Application of Brownian motion to the equation of Kolmogorov-PetrovskijPiskunov, Comm. Pure Appl. Math. 28 (1975), 323-331.

9. H. ENGLER, Relations between traveling wave solutions of quasilinear parabolic equations, Proc. Amer. Math. Soc. 93 (1985), 297-302.

10. J. SMOLLER, Shock waves and reaction diffusion equations (Springer-Verlag, 1982).

LEHRSTUHL FÚR BIOMATHEMATIK

UNIVERSITÄT TÚBINGEN

AUF DER MORGENSTELLE 10

D-7400 TUBINGEN

West Germany 